\title{
Colorectal Cancer Risk Factors and Screening Among the Uninsured of Tampa Bay: A Free Clinic Study
}

\author{
Ethan Y. Song ${ }^{1}$; Justin Swanson, $\mathrm{MPH}^{2}$; Artish Patel ${ }^{1}$; Madeline MacDonald, $\mathbf{M S}^{1}$; \\ Alexandra Aponte ${ }^{3}$; Noura Ayoubi, MD ${ }^{1}$; Lucy Guerra, MD ${ }^{4}$; Eduardo Gonzalez, MD ${ }^{5}$; \\ Rahul Mhaskar, $\mathrm{PhD}^{4}$; Abu-Sayeef Mirza, MD ${ }^{4}$
}

\begin{abstract}
Accessible Version: www.cdc.gov/pcd/issues/2021/20_0496.htm
Suggested citation for this article: Song EY, Swanson J, Patel A, MacDonald M, Aponte A, Ayoubi N, et al. Colorectal Cancer Risk Factors and Screening Among the Uninsured of Tampa Bay: A Free Clinic Study. Prev Chronic Dis 2021;18:200496. DOI: https://doi.org/10.5888/pcd18.200496.
\end{abstract}

\section{PEER REVIEWED}

\section{Summary}

What is already known on this topic?

Patients with low socioeconomic status have a high likelihood of developing colorectal cancer (CRC) due to associated risk factors and lower reported rates of screening.

\section{What is added by this report?}

This study is among the first to analyze the prevalence of CRC risk factors and screening rates of low-income and uninsured patients at free clinics in Florida.

What are the implications for public health practice?

Community-based health centers and free medical clinics are uniquely positioned to treat and care for this vulnerable population through the development of sustainable and cost-effective primary and secondary prevention strategies.

\section{Abstract}

\section{Introduction}

Uninsured patients with low socioeconomic status are at high risk for developing colorectal cancer (CRC), and data on risk factors and prevalence of CRC in this population are limited. The purpose of this study was to assess the risk factors for CRC in uninsured patients from free clinics in the Tampa Bay area of Florida.

\begin{abstract}
Methods
We conducted a retrospective cohort study among patients 50 years or older who were provided service at 9 free clinics in the Tampa Bay area between 2016 and 2018. Demographics, chronic disease characteristics, and screening data were collected via a query of paper and electronic medical records.
\end{abstract}

\section{Results}

Of the 13,982 patients seen, $5,139(36.8 \%)$ were aged 50 years or older. Most were female (56.8\%), non-Hispanic White (41.1\%), and unemployed (54.9\%). Patients with CRC screening were more likely to be employed compared with patients without CRC screening $(54.4 \%$ vs $44.4 \%, P=.01)$. Within the cohort, 725 (22.7\%) patients were active smokers, $771(29.2 \%)$ patients currently consumed alcohol, and 23 patients $(0.4 \%)$ had a history of inflammatory bowel disease. Patients had a median body mass index of 29.4 (interquartile range, $25.4-34.2) \mathrm{kg} / \mathrm{m}^{2}$, and 1,455 $(28.3 \%)$ had diabetes. Documented CRC screening was found among $341(6.6 \%)$ patients.

\section{Conclusion}

Uninsured patients had a high prevalence of CRC risk factors but a low reported screening rate for CRC. Free clinics are uniquely positioned to provide patients at high risk for CRC with strategies to decrease their risk and to be screened for CRC.

\section{Introduction}

Colorectal cancer (CRC) is the third leading cause of cancerrelated deaths in the United States (1) and is most prevalent in patients aged 50 years or older. Despite significant strides in overall cancer survival, several factors such as low income, lack of insurance, and being in a racial or ethnic minority group prevent many Americans from receiving optimal care (1-3). 
Patients with low socioeconomic status have a higher likelihood of developing CRC because of associated risk factors such as alcohol intake, obesity, and smoking (4). Another social determinant of health associated with poor outcomes in patients is lack of health insurance (2). It is well documented that cancer screening rates are lowest in people without health insurance, which leads to high numbers of late-stage cancers (5-7). Patients with Medicaid or those who are uninsured are more likely to have metastatic disease as well as lower rates of definitive surgery and resection (8). Furthermore, patients of racial and ethnic minority groups experience higher incidence and mortality rates of CRC compared with White patients $(3,6)$.

Community-based health centers $(\mathrm{CHC})$ and free medical clinics (FMC) provide primary care services to a large proportion of underinsured and uninsured individuals. They serve as first-line care for the prevention and management of many CRC risk factors such as diet, smoking, alcohol use, and type 2 diabetes (9). However, data on the burden of CRC risk factors in patients of these clinics are limited. The purpose of this study was to assess the prevalence of known risk factors and screening rates of CRC in low-income and uninsured patients of free clinics around Tampa Bay, Florida.

\section{Methods}

We included all uninsured patients served at 9 free clinics in the Tampa Bay area of Florida from January 1, 2016, through December 31, 2018, in this retrospective cohort study. We included patients aged 50 years or older served at any point during the study period, on the basis of US Preventive Services Task Force guidelines that recommend screening for CRC starting at age 50 because of the increased risk of colorectal cancer in this age group (10). We obtained data from paper and electronic medical records and used REDCap software for analyses (11). We compared patients who had documented CRC screening and those who did not by socioeconomic variables (ie, age, sex, race/ethnicity, employment status) and known CRC risk factors, including biometrics (ie, weight and body mass index), alcohol or tobacco use, and comorbidities (ie, diabetes and inflammatory bowel disease [IBD]). We present numeric variables as median (interquartile range [IQR]) and categorical variables as number (\%). We used Mann-Whitney-Wilcoxon tests for numeric variables and $\chi^{2}$ tests for categorical variables; missing values were not included in tests of significance. Significance was set at $P<.05$.

All participating clinics consented to the use of their data. This study was approved by the University of South Florida institutional review board.

\section{Results}

Of the 13,982 patients seen during the study period, $5,139(36.8 \%)$ were aged 50 years or older and included for further analysis (Table 1). Of those with nonmissing demographic data, most were female $(\mathrm{n}=2,896,56.8 \%)$ and unemployed $(\mathrm{n}=1,327,54.9 \%)$, and nearly equal proportions were non-Hispanic White $(\mathrm{n}=1,649$, $41.1 \%)$ and Hispanic of any race $(n=1,639,40.8 \%)$. Of those who reported their smoking status, $725(22.7 \%)$ were active smokers, and 594 (18.6\%) were past smokers (Table 2). Current and past smokers reported a median history of 15 pack-years (IQR, 5-35 pack-years). Of those who reported their history of alcohol consumption, 771 (29.2\%) were active consumers, and $192(7.3 \%)$ were past consumers. The median body mass index (BMI, $\mathrm{kg} / \mathrm{m}^{2}$ ) of patients was 29.4 (IQR, 25.4-34.2). The sample included 1,455 $(28.3 \%)$ patients with diabetes and $23(0.4 \%)$ patients with IBD.

Of all patients, 341 (6.6\%) had a documented CRC screening. Patients with a documented CRC screening were more likely to be employed that those without a screening $(54.4 \%$ vs $44.4 \%, P=$ .01) (Table 1). Patients who had a CRC screening were more likely than those without screening to be active (39.9\% vs $28.0 \%$ ) or past $(10.7 \%$ vs $6.9 \%, P<.001)$ consumers of alcohol (Table 2$)$. Diabetes was more prevalent among patients who received CRC screening than those without $(33.1 \%$ vs $28.0 \%, P=.047)$. IBD was more prevalent among patients with a documented CRC screening than among those without $(1.8 \%$ vs $0.4 \%, P<.001)$.

\section{Discussion}

We found a high prevalence of CRC risk factors among uninsured patients in Tampa Bay's free clinics. More than half of the patients were unemployed and consisted of a largely Hispanic population. We also found a $28.3 \%$ prevalence of diabetes and a median BMI of 29.4, suggesting the continued need for management of chronic health conditions.

The prevalence of several known modifiable risk factors for CRC, including smoking, alcohol usage, poor diet, obesity, and lack of physical activity, is higher in low socioeconomic populations (12-14). Hereditary and personal factors associated with CRC include type 2 diabetes, chronic IBD, and family history of CRC (15-18), and many of these risk factors are seen in higher rates within racial and ethnic minority groups (19). A meta-analysis of 29 articles by Luo and colleagues found that type 2 diabetes was associated with a relative risk of 1.37 (95\% CI, 1.28-1.42) of developing CRC (15). Notably, previous epidemiologic studies show that Hispanics have a high prevalence of overweight and type 2 diabetes $(20,21)$.

\footnotetext{
The opinions expressed by authors contributing to this journal do not necessarily reflect the opinions of the U.S. Department of Health and Human Services, the Public Health Service, the Centers for Disease Control and Prevention, or the authors' affiliated institutions.
} 
Patients who are at high risk for CRC and meet US Preventive Services Task Force guidelines are recommended to have routine CRC screening. However, CRC screening compliance remains a challenge in uninsured patients. Shapiro et al reported that $40 \%$ of Americans aged 50-75 years had not received recommended CRC screening and that the percentage was higher among those without insurance (80\%) (22). Another study by Mojica et al reported that cancer screening rates for Latina women are lower than for nonLatino White women (23). CRC screening rates have been historically lower among Hispanic individuals compared with those who are non-Hispanic White (24). Our results are consistent with the literature, as our patient population was predominantly Hispanic and CRC screening was low, with only $6.6 \%$ of patients undergoing routine screening. Additionally, we found that unemployed patients were more likely not to have CRC screening, emphasizing the need for additional resources or better screening strategies for this population. We have previously reported that epidemiologic estimates may be affected by barriers to health care access, such as transportation, work leave, and the severity of disease (25). Furthermore, although colonoscopy is the gold standard for CRC screening, it is expensive. Socioeconomic status may affect providers' prescription patterns as well as patient compliance. Cheaper alternatives such as the fecal immunochemical test (FIT) and fecal occult blood test (FOBT) can be offered, but these sometimes result in false positive test results (26).

CHCs and FMCs are uniquely positioned to reduce CRC burden because of the large proportion of underinsured and uninsured individuals they serve. Studies show that having a routine source of care is a predictor of CRC test use in these populations $(27,28)$, and several community interventions to increase CRC screening in uninsured patient populations have been successful (29-32). A study by Lairson et al used community health workers, video interventions, or both to increase awareness for colon cancer screening in low-income, uninsured Hispanic patients in El Paso, Texas. These interventions achieved screening rates between $75 \%$ and $87 \%$ compared with $10 \%$ in the comparison group (30). A program for uninsured patients in South Carolina found that FIT screening was more fiscally appropriate for a state's budget and also an effective choice compared with colonoscopy (29). Patients may experience barriers to access to care, such as lack of transportation, so mail-in FOBT can be used to promote screening in low-income populations (31). One of our contributing clinics in Florida has documented screening rates as high as $64 \%$ with the help of a dedicated gastroenterologist and a partnership with the Colon Cancer Alliance (32).

Our study has several limitations, including its retrospective nature and potential for selection bias. Other barriers to health care utilization may exist and may be differentially distributed in the unin- sured population, so our study sample may not be representative of the uninsured population at large. Because the clinics operate independently and have different patient health recording methods, we could not collect and analyze certain data, such as diet and exercise. Study variables often contained large numbers of missing values, which could introduce bias beyond that which occurs with the collection of administrative data. Patients who received CRC screening had lower proportions of missing data on risk factors. Patient or provider knowledge of these risk factors may have increased the likelihood of CRC screening (ie, the presence of multiple risk factors was an apparent requisite for screening), and patients with CRC screening may have been more engaged with their primary care center in general. Another consideration is that although our original study focused on a spectrum of chronic diseases, our database did not capture the entire granularity of screening methods (eg, colonoscopy, FIT, FOBT) for CRC.

Nevertheless, our study is among the few that have reported the burden of risk factors and screening rates for CRC in FMCs in Florida (32). Overall, our study further elucidates the disparity of risk factors and $\mathrm{CRC}$ burden in the low-income and uninsured population. Because CHCs and FMCs are uniquely positioned to treat and care for this population, our findings should encourage the development of sustainable and cost-effective primary and secondary prevention strategies for this vulnerable group.

\section{Conclusion}

Low-income and uninsured patients of the free clinics in Tampa Bay are at a higher risk of developing CRC because of higher rates of predisposing comorbidities. Continued management of risk factors and increased screening efforts should be made for this vulnerable population. Subsidized screening, including FIT tests and colonoscopies, would strongly benefit these high-risk patients and increase the resources available at free clinics for such preventive measures.

\section{Acknowledgments}

We thank the University of South Florida Office of Research, Innovation, and Scholarly Endeavors for providing a stipend to several medical students who participated in the data collection process as part of a summer research experience. We also thank the clinic directors and staff members at the 9 free clinics where the data were obtained. Finally, we thank our team of undergraduate, graduate, and medical student volunteers, Dr Laurie Woodard, Kevin Casey, Shirley Smith, and the University of South Florida Office of Research, Innovation, and Scholarly Endeavors for providing expertise paramount to the success of this project. No

The opinions expressed by authors contributing to this journal do not necessarily reflect the opinions of the U.S. Department of Health and Human Services, the Public Health Service, the Centers for Disease Control and Prevention, or the authors' affiliated institutions. 
funding was received for the completion of this study (no. Pro00023920), and no copyrighted materials or tools were used in this research.

\section{Author Information}

Corresponding Author: Ethan Y. Song, University of South Florida, Morsani College of Medicine, 12901 Bruce B. Downs Blvd, Tampa, FL 33612. Telephone: 858-243-6224. Email: esong@usf.edu.

Author Affiliations: ${ }^{1}$ University of South Florida, Morsani College of Medicine, Tampa, Florida. ${ }^{2}$ University of South Florida, College of Public Health, Tampa, Florida. ${ }^{3}$ University of South Florida, Tampa, Florida. ${ }^{4}$ USF Morsani College of Medicine, Department of Internal Medicine, Tampa, Florida. ${ }^{5}$ USF Morsani College of Medicine, Department of Family Medicine, Tampa, Florida.

\section{References}

1. Siegel RL, Miller KD, Jemal A. Cancer statistics, 2020. CA Cancer J Clin 2020;70(1):7-30.

2. Roetzheim RG, Pal N, Gonzalez EC, Ferrante JM, Van Durme DJ, Krischer JP. Effects of health insurance and race on colorectal cancer treatments and outcomes. Am J Public Health 2000;90(11):1746-54.

3. DeSantis CE, Miller KD, Goding Sauer A, Jemal A, Siegel RL. Cancer statistics for African Americans, 2019. CA Cancer J Clin 2019;69(3):211-33.

4. Zhao J, Zhu Y, Wang PP, West R, Buehler S, Sun Z, et al. Interaction between alcohol drinking and obesity in relation to colorectal cancer risk: a case-control study in Newfoundland and Labrador, Canada. BMC Public Health 2012;12(1):94.

5. Centers for Disease Control and Prevention (CDC). Vital signs: colorectal cancer screening test use - United States, 2012. MMWR Morb Mortal Wkly Rep 2013;62(44):881-8.

6. Eberth JM, Thibault A, Caldwell R, Josey MJ, Qiang B, Peña E, et al. A statewide program providing colorectal cancer screening to the uninsured of South Carolina. Cancer 2018; 124(9):1912-20.

7. Farkas DT, Greenbaum A, Singhal V, Cosgrove JM. Effect of insurance status on the stage of breast and colorectal cancers in a safety-net hospital. Am J Manag Care 2012;18(5 Spec No 2):65-70.

8. Pulte D, Jansen L, Brenner H. Disparities in colon cancer survival by insurance type: a population-based analysis. Dis Colon Rectum 2018;61(5):538-46.
9. Nadkarni MM, Philbrick JT. Free clinics and the uninsured: the increasing demands of chronic illness. J Health Care Poor Underserved 2003;14(2):165-74.

10. US Preventive Services Task Force. Colorectal cancer: screening; 2016. https://www.uspreventiveservicestaskforce .org/uspstf/recommendation/colorectal-cancer-screening. Accessed February 3, 2021.

11. Harris PA, Taylor R, Thielke R, Payne J, Gonzalez N, Conde JG. Research electronic data capture (REDCap) — a metadatadriven methodology and workflow process for providing translational research informatics support. J Biomed Inform 2009;42(2):377-81.

12. Sauer AG, Siegel RL, Jemal A, Fedewa SA. Updated review of prevalence of major risk factors and use of screening tests for cancer in the United States. Cancer Epidemiol Biomarkers Prev 2017;26(8):1192-208.

13. Doubeni CA, Major JM, Laiyemo AO, Schootman M, Zauber AG, Hollenbeck AR, et al. Contribution of behavioral risk factors and obesity to socioeconomic differences in colorectal cancer incidence. J Natl Cancer Inst 2012;104(18):1353-62.

14. Cao Y, Willett WC, Rimm EB, Stampfer MJ, Giovannucci EL. Light to moderate intake of alcohol, drinking patterns, and risk of cancer: results from two prospective US cohort studies. BMJ 2015;351:h4238.

15. Luo S, Li JY, Zhao LN, Yu T, Zhong W, Xia ZS, et al. Diabetes mellitus increases the risk of colorectal neoplasia: an updated meta-analysis. Clin Res Hepatol Gastroenterol 2016; 40(1):110-23.

16. Butterworth AS, Higgins JP, Pharoah P. Relative and absolute risk of colorectal cancer for individuals with a family history: a meta-analysis. Eur J Cancer 2006;42(2):216-27.

17. Lutgens MW, van Oijen MG, van der Heijden GJ, Vleggaar FP, Siersema PD, Oldenburg B. Declining risk of colorectal cancer in inflammatory bowel disease: an updated metaanalysis of population-based cohort studies. Inflamm Bowel Dis 2013;19(4):789-99.

18. De Bruijn KM, Arends LR, Hansen BE, Leeflang S, Ruiter R, van Eijck $\mathrm{CH}$. Systematic review and meta-analysis of the association between diabetes mellitus and incidence and mortality in breast and colorectal cancer. Br J Surg 2013; 100(11):1421-9.

19. Singh GK, Daus GP, Allender M, Ramey CT, Martin EK, Perry C, et al. Social determinants of health in the United States: addressing major health inequality trends for the nation, 1935-2016. Int J MCH AIDS 2017;6(2):139-64.

20. Caballero AE. Type 2 diabetes in the Hispanic or Latino population: challenges and opportunities. Curr Opin Endocrinol Diabetes Obes 2007;14(2):151-7.

The opinions expressed by authors contributing to this journal do not necessarily reflect the opinions of the U.S. Department of Health and Human Services, the Public Health Service, the Centers for Disease Control and Prevention, or the authors' affiliated institutions. 
21. Bhupathiraju SN, Hu FB. Epidemiology of obesity and diabetes and their cardiovascular complications. Circ Res 2016;118(11):1723-35.

22. Shapiro JA, Klabunde CN, Thompson TD, Nadel MR, Seeff LC, White A. Patterns of colorectal cancer test use, including CT colonography, in the 2010 National Health Interview Survey. Cancer Epidemiol Biomarkers Prev 2012; 21(6):895-904.

23. Mojica CM, Flores B, Ketchum NS, Liang Y. Health care access, utilization, and cancer screening among low-income Latina women. Hisp Health Care Int 2017;15(4):160-5.

24. Colorectal cancer facts and figures, 2020-2022. Atlanta (GA): American Cancer Society; 2020.

25. Rahman S, Mirza AS, Stenback J, Green S, Mayers Y, Iranmanesh $\mathrm{E}$, et al. Burden of chronic conditions among patients from free clinics: a retrospective chart review of 2015. J Health Care Poor Underserved 2018;29(3):1011-26.

26. Hewitson P, Glasziou P, Irwig L, Towler B, Watson E. Screening for colorectal cancer using the faecal occult blood test, Hemoccult. Cochrane Database Syst Rev 2007; 2007(1):CD00126.

27. Jandorf L, Ellison J, Villagra C, Winkel G, Varela A, Quintero-Canetti Z, et al. Understanding the barriers and facilitators of colorectal cancer screening among low income immigrant Hispanics. J Immigr Minor Health 2010; 12(4):462-9.

28. Hughes AG, Watanabe-Galloway S, Schnell P, Soliman AS. Rural-urban differences in colorectal cancer screening barriers in Nebraska. J Community Health 2015;40(6):1065-74.

29. van der Steen A, Knudsen AB, van Hees F, Walter GP, Berger FG, Daguise VG, et al. Optimal colorectal cancer screening in states' low-income, uninsured populations - the case of South Carolina. Health Serv Res 2015;50(3):768-89.

30. Lairson DR, Kim J, Byrd T, Salaiz R, Shokar NK. Costeffectiveness of community interventions for colorectal cancer screening: low-income Hispanic population. Health Promot Pract 2018;19(6):863-72.

31. Jean-Jacques M, Kaleba EO, Gatta JL, Gracia G, Ryan ER, Choucair BN. Program to improve colorectal cancer screening in a low-income, racially diverse population: a randomized controlled trial. Ann Fam Med 2012;10(5):412-7.

32. Zein K, Courtney U, Yohan P, Brian A, Erin A, Felicia O, et al. A model for colon cancer screening at a free community clinic. Journal of Student-Run Clinics 2018;4(1).

The opinions expressed by authors contributing to this journal do not necessarily reflect the opinions of the U.S. Department of Health and Human Services, the Public Health Service, the Centers for Disease Control and Prevention, or the authors' affiliated institutions. 


\section{Tables}

Table 1. Demographics of Uninsured Patients Serviced at 9 Free Clinics in Tampa Bay, Florida, 2016-2018

\begin{tabular}{|c|c|c|c|c|}
\hline Demographic Characteristic & All Patients $(\mathrm{N}=5,139)$ & No CRC Screening $(n=4,798)$ & CRC Screening $(n=341)$ & $P$ Value \\
\hline Age, median, y (IQR) & $58(54-62)$ & $58(54-62)$ & $58(54-61)$ & .35 \\
\hline \multicolumn{5}{|l|}{ Sex } \\
\hline Male & $2,206(43.2)$ & $2,074(43.6)$ & $132(38.7)$ & \multirow{3}{*}{.0} \\
\hline Female & $2,896(56.8)$ & $2,687(56.4)$ & $209(61.3)$ & \\
\hline Missing & $37(0)$ & $37(0)$ & 0 & \\
\hline \multicolumn{5}{|l|}{ Race/ethnicity } \\
\hline White & $1,649(41.1)$ & $1,504(40.6)$ & $145(47.1)$ & \multirow{6}{*}{.0} \\
\hline Black & $507(12.6)$ & $465(12.5)$ & $42(13.6)$ & \\
\hline Asian & $194(4.8)$ & $183(4.9)$ & $11(3.6)$ & \\
\hline Hispanic, all races & $1,639(40.8)$ & $1,533(41.4)$ & $106(34.4)$ & \\
\hline Other & $26(0.7)$ & $22(0.6)$ & $4(1.3)$ & \\
\hline Missing & $1,124(0)$ & $1,091(0)$ & $33(0)$ & \\
\hline \multicolumn{5}{|l|}{ Employment } \\
\hline Employed & 1,091 (45.1) & $998(44.4)$ & $93(54.4)$ & \multirow{3}{*}{.01} \\
\hline Unemployed & $1,327(54.9)$ & $1,249(55.6)$ & $78(45.6)$ & \\
\hline Missing & $2,721(0)$ & $2,551(0)$ & $170(0)$ & \\
\hline
\end{tabular}

Abbreviations: CRC, colorectal cancer: IQR, interquartile range (quartile 1-quartile 3).

${ }^{a}$ Values are no. (\%) unless otherwise indicated. 
Table 2. Clinical Colorectal Cancer Risk Factors of Uninsured Patients Serviced at 9 Free Clinics in Tampa Bay, Florida, 2016-2018 ${ }^{\mathrm{a}}$

\begin{tabular}{|c|c|c|c|c|}
\hline Risk Factor & All Patients $(N=5,139)$ & No CRC Screening $(n=4,798)$ & CRC Screening $(n=341)$ & $P$ Value \\
\hline \multicolumn{5}{|l|}{ BMI, median, $\mathrm{kg} / \mathrm{m}^{2}$ (IQR) } \\
\hline Sample & $29.4(25.4-34.2)$ & $29.3(25.4-34.2)$ & $29.7(25.6-34.4)$ & \multirow{2}{*}{.94} \\
\hline Missing & $1,546(0)$ & $1,527(0)$ & $19(0)$ & \\
\hline \multicolumn{5}{|l|}{ Smoking status } \\
\hline Active & $725(22.7)$ & $656(22.6)$ & $69(23.0)$ & \multirow{4}{*}{.09} \\
\hline Past & $594(18.6)$ & $525(18.1)$ & $69(23.0)$ & \\
\hline Never & $1,879(58.8)$ & $1,717(59.2)$ & $162(54.0)$ & \\
\hline Missing & $1,941(0)$ & $1,900(0)$ & $41(0)$ & \\
\hline \multicolumn{5}{|l|}{ Alcohol consumption } \\
\hline Active & $771(29.2)$ & $663(28.0)$ & $108(39.9)$ & \multirow{4}{*}{$<.001$} \\
\hline Past & $192(7.3)$ & $163(6.9)$ & $29(10.7)$ & \\
\hline Never & $1,676(63.5)$ & $1,542(65.1)$ & $134(49.4)$ & \\
\hline Missing & $2,500(0)$ & $2,430(0)$ & $70(0)$ & \\
\hline \multicolumn{5}{|l|}{ Chronic illness } \\
\hline Diabetes & $1,455(28.3)$ & $1,342(28.0)$ & $113(33.1)$ & .047 \\
\hline Inflammatory bowel disease & $23(0.4)$ & $17(0.4)$ & $6(1.8)$ & $<.001$ \\
\hline
\end{tabular}

Abbreviations: CRC, colorectal cancer; BMI, body mass index; IQR, interquartile range.

${ }^{a}$ Values are no. (\%) unless otherwise indicated. 